\title{
Heartbeat: higher risk of COVID-19 infection in younger patients with cardiovascular disease
}

doi:10.1136/heartjnl-2021-319052

Catherine M Otto

Cardiovascular disease (CVD) is a major risk factor for adverse outcomes with COVID-19 infection. Concerns raised early in the pandemic that reninangiotensin system (RAS) blockade by treatment with angiotensin converting enzyme (ACE) inhibitors or angiotensin receptor blockers might increase that risk have not been supported by observational studies and meta-analyses. ${ }^{12}$ As Simon and Danchin ${ }^{3}$ point out, 'jumping to therapeutic conclusions on the sole basis of pathophysiological or experimental considerations is hazardous. Nature can be tricky, and pathways that are blocked by a therapeutic intervention are often short-circuited by other, secondary, pathways that will result in the lack of clinical effect of the said therapeutic intervention. Here, in spite of the recognised interference between ACE2 and SARS-CoV-2, and of the possible link between RAS blockers and ACE2 in humans, these medications appeared clinically neutral.'

Age also is a major risk factor for COVID-19 infection, with previous analyses treating age as a potential confounder. While this approach often is appropriate, treating age as a confounder might obscure any differences in risk among younger and older patients with CVD. In order to examine the effect of age on the risk of fatal outcomes with COVID-19 infection in patients with CVD, Bae and colleagues ${ }^{4}$ performed a meta-analysis of 51 studies including a total of over 48000 patients with a confirmed COVID-19 infection. Fatal outcomes with COVID-19 infection were strongly associated with diabetes, hypertension and CVD across all age groups (hypertension: OR 2.50, 95\% CI 2.15 to 2.90 ; diabetes: $2.25,95 \% \mathrm{CI}$ 1.89 to 2.69 ; CVD: 3.11 , 95\% 2.55 to $3.79)$. When stratified by age, younger patients (age $<50$ years) had a lower prevalence of cardiovascular comorbidities than older patients (age $>60$ years). However, fatality rates with COVID-19 infection were higher (compared with

Correspondence to Professor Catherine M Otto, Division of Cardiology, University of Washington, Seattle, WA 98195, USA; cmotto@uw.edu
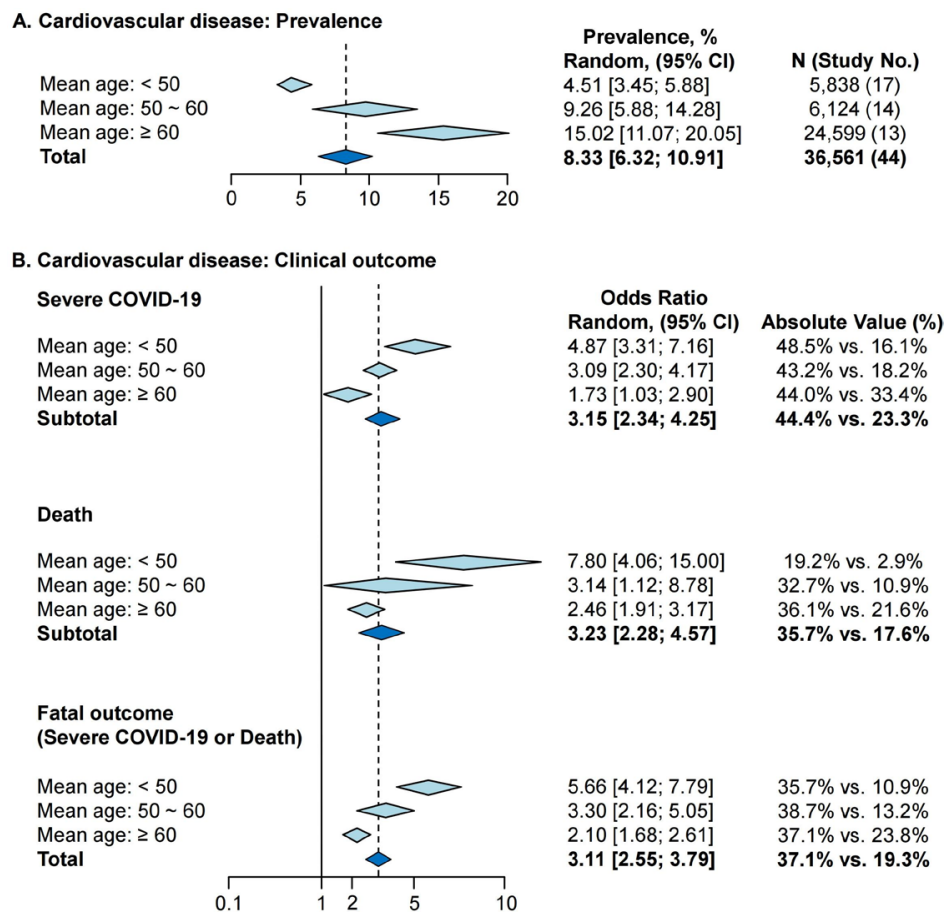

Figure 1 Prevalence of cardiovascular disease and clinical outcomes by age.

older patients) in those younger patients who did have diabetes, hypertension or CVD (figure 1). This study level metaanalysis has several limitations, and the pathophysiological basis for these agerelated differences is not clear, but these findings draw attention to the possibility that adults $<50$ years old with established CVD or CVD risk factors may be at an even higher risk of adverse outcomes with COVID-19 infection, despite their young age.

A review article in this issue of Heart summarises the data on the relationship between socioeconomic status and cardiovascular health during the COVID-19 pandemic. $^{5}$ Potentially some of these factors account for the high risk

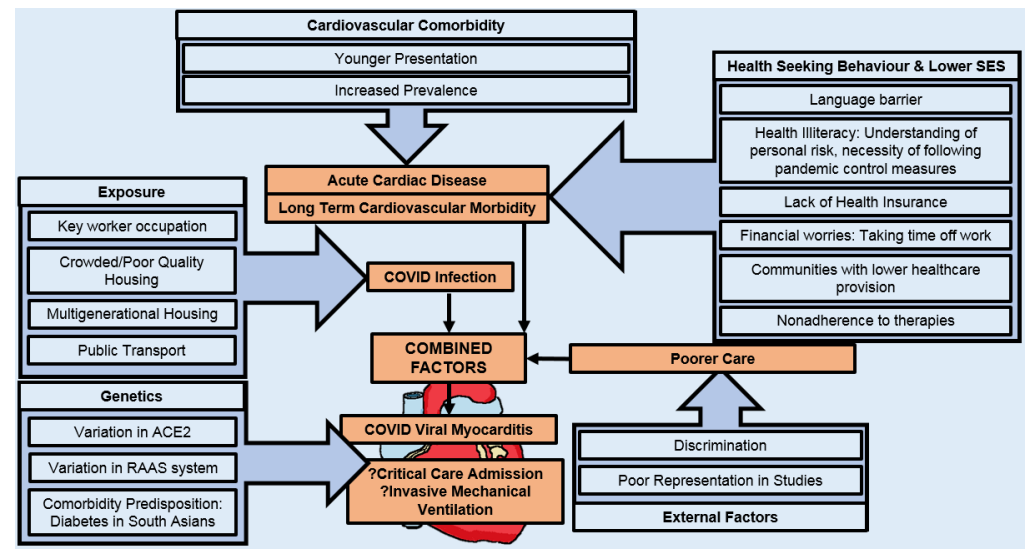

Figure 2 Predisposing factors to poor outcomes in black, Asian and minority ethnic group. RAAS, renin-angiotensin-aldosterone system; SES, socioeconomic status. 


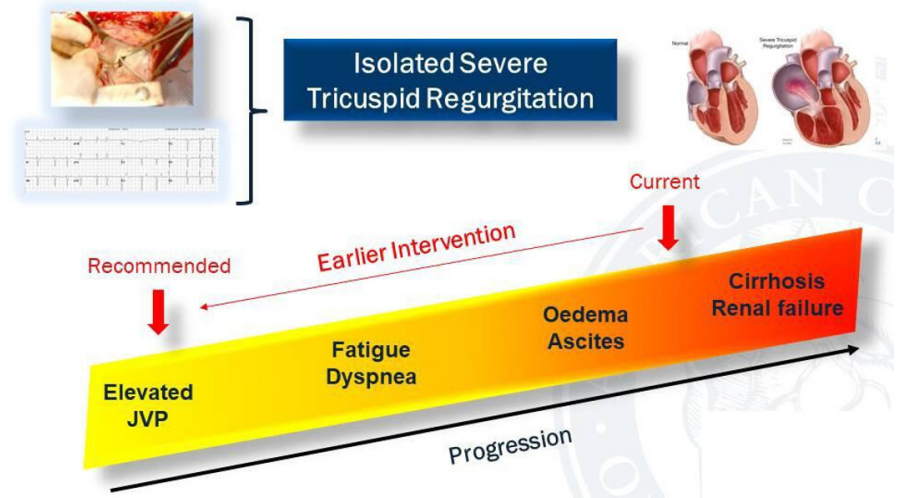

Figure 3 Timing of intervention in isolated severe tricuspid regurgitation. JVP, jugular venous pressure. Currently, patients are typically operated on at an advanced stage of disease with cirrhosis and renal failure with high operative mortality. Earlier intervention with onset of symptoms might improve outcomes. In isolated severe tricuspid regurgitation, intervention should be considered in the symptomatic patient once jugular venous pressure elevation is noted as a reflection of right atrial pressure elevation.

of infection in younger patients with CVD (figure 2). The authors go on to propose measures to reduce or blunt the effect of socioeconomic disparities on patients with CVD, which iinclude use of screening tools by healthcare professionals to identify high-risk individuals, improved interventions in high-risk individuals, and provision of appropriate patient education.

Also in this issue of Heart, Park and colleagues report the outcomes of 238 patients undergoing isolated tricuspid valve surgery for severe tricuspid regurgitation (TR). ${ }^{6}$ Over a 4-year follow-up, the risk of death or heart transplantation was high $(>20 \%)$ with predictors of outcome including age, haemoglobin level, bilirubin, TR severity and right atrial pressure. There was a trend favouring valve replacement over repair in those with an annulus diameter $>44 \mathrm{~mm}$. Even so, in an editorial Reddy et $a l^{7}$ recommend that 'it is reasonable to consider surgery for symptomatic severe TR that is either (1) primary to intrinsic valve pathology or (2) isolated in the absence of pulmonary hypertension or cardiomyopathy.' They also suggest 'it appears prudent to consider intervention before the development of right heart myocardial abnormalities or endorgan damage' (figure 3).

The association of mitral valve prolapse (MVP) and ventricular arrhythmias was

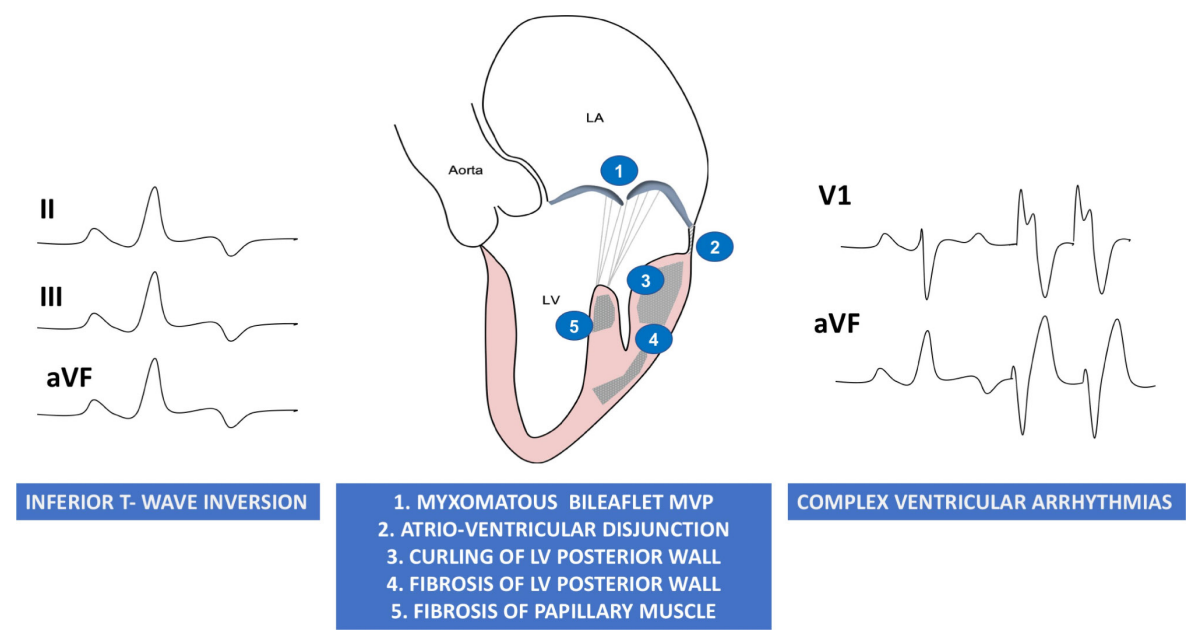

Figure 4 Stigmata of arrhythmic MVP. ECG repolarisation abnormalities, more commonly T-wave inversion in inferior leads (left panel). Morphofunctional abnormalities including myxomatous bileaflet MVP, atrioventricular disjunction, curling (and hypertrophy) of LV posterior basal wall, fibrosis of LV posterior wall and papillary muscles (central panel). Complex ventricular arrhythmias, more frequently with right bundle branch block morphology (right panel). LA, left atrium; LV, left ventricular; MVP, mitral valve prolapse.

addressed in a study of 610 MVP patients, of whom $11 \%$ had symptomatic arrhythmias-mostly non-sustained ventricular tachycardia (VT) or frequent premature ventricular beats but with three patients having sustained VT and another three with ventricular fibrillation. ${ }^{8}$ Independent predictors of ventricular arrhythmias were female sex, increased mitral annular diameter, lower left ventricular global longitudinal strain and prolonged mechanical dispersion. In an editorial, Cipriani and Bauce' propose that 'MVPs are not all the same, and that the 'arrhythmic MVP' is a peculiar clinical entity, characterised by specific mitral valve apparatus abnormalities, such as myxomatous, redundant and prolapsing leaflets, mitral annular disjunction (MAD), replacement fibrotic changes in papillary muscles and basal myocardium' (figure 4).

The Education in Heart $\operatorname{article}^{10}$ in this issue presents basic concepts in the diagnosis and management of atrial fibrillation with attention to the issues of overcoming barriers to anticoagulation, risk stratification for both thromboembolic and bleeding, and indications for antithrombotic therapy in specific patients groups, including surgical patients, those undergoing cardiovascular interventions and those with renal dysfunction or on dialysis.

Funding The authors have not declared a specific grant for this research from any funding agency in the public, commercial or not-for-profit sectors.

Competing interests None declared.

Patient and public involvement Patients and/or the public were not involved in the design, or conduct, or reporting, or dissemination plans of this research.

Patient consent for publication Not required.

Provenance and peer review Commissioned; internally peer reviewed.

(C) Author(s) (or their employer(s)) 2021. No commercial re-use. See rights and permissions. Published by BMJ.

\section{Check for updates}

To cite Otto CM. Heart 2021;107:345-347.

Heart 2021;107:345-347.

doi:10.1136/heartjnl-2021-319052

ORCID iD

Catherine M Otto http://orcid.org/0000-0002-05279392

\section{REFERENCES}

1 Hippisley-Cox J, Young D, Coupland C, et al. Risk of severe COVID-19 disease with ACE inhibitors and angiotensin receptor blockers: cohort study including 8.3 million people. Heart 2020;106:1503-11.

2 Flacco ME, Acuti Martellucci C, Bravi F, et al. Treatment with ACE inhibitors or ARBs and risk 
of severe/lethal COVID-19: a meta-analysis. Heart 2020;106:1519-24.

3 Simon T, Danchin N. Beware of simple explanations. Heart 2021;107:348-9.

4 Bae S, Kim SR, Kim M-N. Impact of cardiovascular disease and risk factors on fatal outcomes in patients with COVID-19 according to age: a systematic review and meta-analysis. Heart 2021;107:373-80.
5 Naylor-Wardle J, Rowland B, Kunadian V. Socioeconomic status and cardiovascular health in the COVID-19 pandemic. Heart 2021;107:358-65.

6 Park SJ, Oh JK, Kim S-O. Determinants of clinical outcomes of surgery for isolated severe tricuspid regurgitation. Heart 2021;107:403-10.

7 Reddy YNV, Alkhouli M, Nishimura RA. Isolated severe tricuspid regurgitation: an unrecognised and undertreated problem of the forgotten valve. Heart 2021;107:350-2.
8 van Wijngaarden AL, de Riva M, Hiemstra YL. Parameters associated with ventricular arrhythmias in mitral valve prolapse with significant regurgitation. Heart 2021;107:411-8.

9 Cipriani A, Bauce B. Ventricular arrhythmias in mitral valve prolapse: new explanations for an old problem. Heart 2021;107:353-4.

10 Barra S, Providência R. Anticoagulation in atrial fibrillation. Heart 2021:107:419-27. 